\section{The chocolate box problem}

\section{Peter Bryant}

Autism: Explaining the Enlgma. By Uta Frith. Basil Blackwell: 1989. Pp.204. Hbk £25, \$19.95; pbk £8.95.

THE number of autistic children is tiny (about 4 in 10,000). Yet their plight has always attracted a great deal of attention because the nature of their difficulties poses an intriguing puzzle.

Autistic children have severe social and emotional problems, and they have some striking intellectual difficulties as well. This combination of problems is unusual in itself, but it seems even more curious when one considers the uneveness of their intellectual abilities. They tend to fail dismally in some intellectual tasks, and yet have little difficulty in others. Some display remarkable talents - there are autistic children who draw astonishingly accurate and beautiful pictures, and others who have remarkable musical abilities, and yet can barely speak.

The general interest in autism is reflected in the large amount of research that has been done on the subject, but there has never been an accessible and comprehensive account of this work. Uta Frith, who is one of the leading researchers on childhood autism, has now filled this gap. Her exciting and attractively written book can be divided into two parts. In the first she lays out the facts - the odd combination of symptoms, the possible influence of heredity, the social class and gender differences - and thus sets the puzzle. The aim of the rest of the book is to provide the solution.

In fact two solutions are offered, or at any rate two hypotheses. The first is that autistic children are insensitive to pattern. Most children find it easier to remember a meaningful sentence than a list of disconnected words, but that is not true of autistic children. They are not helped by meaning. They also often fail to spot repetitive patterns in perceptual displays, though such patterns are perfectly obvious to other children. Sometimes the attention that autistic children pay to parts at the expense of the whole actually helps them. They are better than other children at spotting an abstract shape which is hidden within a larger meaningful figure. So Uta Frith argues that their world is fragmented and that it lacks meaning.

Her second theme is that autistic children find it particularly hard to work out what other people are thinking and feeling. She and her colleagues have shown, to take but one example, that autistic children manage perfectly well when asked to arrange some pictures into a sequence that tells a story about a balloon flying away and bursting on a tree, or about someone going to a shop and buying sweets. But they are at a loss when the story concerns a boy's disappointment at not finding something which he had put in a box for safe keeping (and which someone else had stolen). When other people's expectations and emotions are different from the autistic child's they are beyond his comprehension.

Uta Frith links this difficulty to another peculiarity of autism - the fact that autistic children tend not to indulge in 'pretend play'. Normal children, when playing, quite often decide, for example, that a banana can play the part of a telephone, but as far as the autistic child is concerned it stubbornly remains a banana. Uta Frith argues that one has to know about pretence to understand that two people can have different beliefs about the same event. If I pretend that an empty chocolate box is full, I know that I may believe one thing and the rest of the world another about the box's contents. According to Uta Frith autistic children do not understand pretence and thus cannot grasp the fact that different people have different feelings and beliefs. As a result many of the most important aspects of social communication elude them completely.

\section{Signal survey}

\section{Gunnar von Heijne}

Protein Targeting. By Anthony P. Pugsley Academic: 1989 . Pp.279. \$45, £28.50.

Protein targeting - that is, the means by which newly made proteins are directed to their final destinations inside or outside the cell - has been among the hottest subjects in molecular and cell biology for more than a decade. Yet it is only with Anthony Pugsley's book that a wellwritten, integrated overview of the field has become available.

Pugsley starts with two introductory chapters on protein traffic in eukaryotic and bacterial cells, and the main experimental techniques used to study these processes. He then takes a deep breath and dives into his subject, following the sometimes tortuous paths of proteins from the cytoplasm through all the intricacies of the secretory pathway or into the depths of nuclei, mitochondria and chloroplasts.

Throughout, the emphasis is on the structure of the routing signals and on how these are decoded by a battery of cytoplasmic, membrane-bound and intraorganellar proteins that ensure the specificity and vectoriality of targeting and transport. Pugsley provides a unified picture, based on the latest results, with cytoplasmic factors stabilizing 'transloca-
This in turn accounts for their social and also for their linguistic difficulties.

Uta Frith's presentation of these two hypotheses is lucid, entertaining and extremely convincing. There is no doubt that the empirical evidence which supports her ideas is the most exciting that there is on the subject of autism. There are a few objections to be made. Autistic children may have a better understanding of pretence than she suggests: some recent work shows that they do take to pretend play if they are prompted to do so. There is also a certain tension between the two hypotheses. For example autistic children easily rearranged pictures into a story about a balloon bursting, and yet according to Uta Frith's ideas about fragmentation it should have been quite difficult for them to do so. Her explanation is that they have difficulty with broad but not with narrow contexts: but how does one decide what is broad?

These are minor quibbles while the book is without doubt a major achievement. It will have an immense influence, and all to the good, on future research on this topic.

Peter Bryant is in the Department of Experimental Psychology, University of Oxford, South Parks Road, Oxford OX1 3UD, UK.

tion-competent' conformations of the newly synthesized proteins, organellebound receptors recognizing their cognate routing signals, integral membrane proteins possibly serving as translocation machines, and 'chaperones' inside the organelles assisting in the final steps of folding and assembly.

Also discussed are the vesicular communication links between the different stages of the secretory pathway - endoplasmic reticulum to Golgi to trans-Golgi network to plasma membrane or lysosome - and the complementary processes of endocytotic uptake of proteins and other molecules from the cells' surroundings.

The text ends with an appendix describing various ways that our knowledge about protein targeting can be put to practical ends: secretion of heterologous proteins by bacterial, fungal and animal cell cultures; herbicide-resistance by manipulation of chloroplast-targeted proteins; and specific targeting of toxins into the endocytic pathway of tumor cells.

As a befitting finale there is an impressive bibliography with some 1,300 references, essentially complete up to and including 1988. Let us hope that the royalties Pugsley can expect from his book will go at least some way towards covering his no doubt exorbitant photocopying bills.

Gunnar von Heijne is in the Department of Molecular Biology, Karolinska Institute Center for Biotechnology, Novum, S-14152 Huddinge, Sweden. 